\title{
Assessment of Technical Condition and Readiness of Pump Units by Diagnostic Methods
}

\author{
M. M. Muhammadiev ${ }^{1 *}, O . Y$. Glovatsky $^{2}, R . R$. Ergashev $^{3}, N . R$. Nasyrova $^{1}$ and \\ F. S. Saidov ${ }^{1}$ \\ ${ }^{1}$ Tashkent State Technical University named after Islam Karimov, Tashkent, Uzbekistan \\ ${ }^{2}$ Research Institute of Irrigation and Water Problems, Tashkent, Uzbekistan \\ ${ }^{3}$ Tashkent Institute of Irrigation and Agricultural Mechanization Engineers, Tashkent, Uzbekistan
}

\begin{abstract}
The article presents the developed methods and models for analyzing the dynamic indicators of pumping units for different time intervals of their state during operation. The main tasks of assessing the technical condition are to identify deviations from design solutions, the presence of damage, defects in pump structures that can cause accidents. The results of the development of a diagnostic system for pumping units at pumping stations are presented. The working conditions of the connecting links of the main pumping and power equipment and its diagnostics are considered. The introduction of the proposed diagnostic system will lead to a reduction in operating costs of pumping units and the cost of their maintenance.
\end{abstract}

\section{Introduction}

The technical condition of irrigation pump units (PU) should reflect the continuity and effective coverage of a given water supply schedule, the cost of pumped water, as well as reliability indicators of units [1-3].

The main purpose of assessing the technical condition of the scientific equipment is to take into account the aging factors of nodes, channels and hydraulic units. This can be done on the basis of diagnostics of the dynamic level of PU elements during operation. After analyzing the data obtained, it will be possible to make a decision on the further use or modernization of the scientific equipment.

To determine the technical condition of the PU, diagnostic and structural parameters are used, which are calculated according to the appropriate algorithms. Parameters calculated directly from characteristic functions (spectra, signals) are called diagnostic parameters. Parameters that are a set of diagnostic parameters are called structural parameters.

\footnotetext{
*Corresponding author: muhammadiyev m@ rambler.ru
} 


\section{Materials and methods}

Assessment of the technical condition and operational safety is the basis for the reconstruction of the hydraulic unit of pumping stations.

The methods of a fundamentally new theoretical approach to the problem of the reliability of the functioning of complex systems are used.

\section{Results}

It is known that in most cases, the "diagnostics" of the technical condition of the scientific equipment is limited to measuring the performance characteristics, namely, the pressure and flow. Moreover, due to the absence or high cost of flow meters, most often, only the pressure $[2,4]$.

The head, therefore, is the main indicator of the unit's performance. However, despite the ease of installation of pressure gauges for its measurement and high accuracy, the absolute value of the pressure without correlating it with the flow rate does not make it possible to assess the true technical condition of the pump. This is due to the fact that the head is highly dependent on the flow rate, which, in turn, is a function of the pump operating mode in a broad sense.

A generalized indicator of the technical condition of the pumping unit consists of three complex parameters: technical condition, technical level and technological process, which comprehensively reflect the properties of the pumping unit during operation.

The versatility of the generalized assessment of the technical condition of the pump unit allows you to compare different types of pumps.

To assess the technical condition, it is most appropriate to use the technical condition indices:

$$
V_{q}=\prod_{i=1}^{n} q_{i}^{a_{i}}
$$

where $-n$ is the number of indicators of the technical condition; $q_{i}-$ is an indicator of the technical condition; $i$-is the weight parameter of the $i$-th indicator of the technical condition. To approximate the flow characteristics of the pumping unit, modern methods are used that use various compactly supported polynomial functions $[4,5]$.

A modern and perfect way to detect defects in the operation of the pumping unit is to diagnose. The authors have developed a new diagnostic system.

A new diagnostic system has been developed.

New diagnostic systems are used at full-scale objects and stands of Tashkent Institute of Irrigation and Agricultural Mechanization, Tashkent State Technical University and Production Association "SUVMASH" (figure 1). 


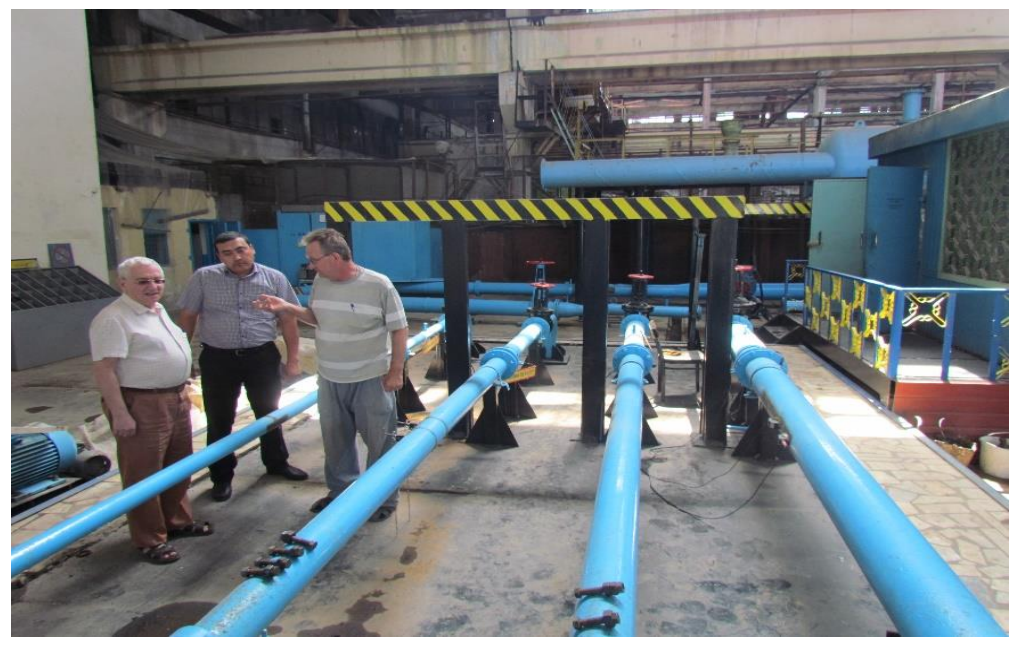

Fig. 1. Research of new diagnostic systems.

The main purpose of PU diagnostics is to predict the possibility of defects in them during operation and to ensure reliable and efficient operation.

Currently, most portable or temporary diagnostic equipment is used to determine the status of pumps. In determining the state of pumping units, the information-theoretic approach is promising.

During the operation of pumping stations, it is possible to detect cavitation and the stage of its development by vibration in modes when, for example, there is cavitation in the pump, but there is still no cavitation breakdown, leading to a sharp drop in pressure and flow at the pump outlet, to detect cavitation and the stage of its development, i.e. perform pump flow diagnostics.

Based on the kinematic scheme shown in figure 2, a laboratory bench was developed to study the technical condition of the pumping unit, where a number of experiments were carried out $[6,7]$.

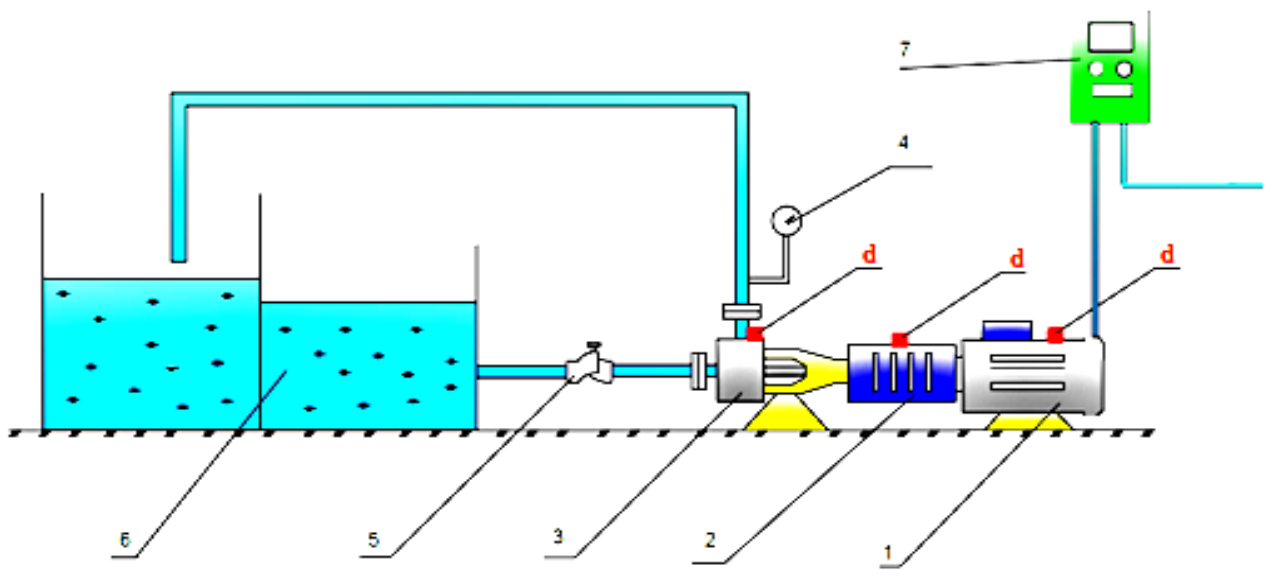

Fig. 2. Kinematic diagram of the pump shaker: 1 -electric motor; 2 -gear; 3 -pump flange; 4 - manometer for measuring water pressure; 5 -tap; 6 - tank for water; 7 - pump rotation control device; $d$ vibration sensors 
The laboratory stand consists of an electric motor, power transmission couplings, pumps, monometers for measuring water pressure, valves for closing and opening water, as well as a device for changing pump parameters and pump speed. The water intake is filled with the required amount of water. To open the water, the valve opens, and the water flows through the pipe to the pumping station. Depending on the parameters, the current state of specific PU nodes is determined.

At present, the diagnostic and control equipment installed on the scientific equipment allows continuous diagnostics of water flow and pressure in pumps, lubricating oil temperature, current and output power of the electric motor. Vibration diagnostics systems, which can lead to emergency situations, are developed and insufficiently applied.

The diagnostic system provides a continuous assessment of the condition of the pumping unit, reducing maintenance costs, their safe and reliable operation and repair. This system automatically detects changes in the pump unit and provides a database of faults and keeps track of the need to solve specific problems.

Studies carried out with PU, which have the same operating conditions, have shown that the vibration level in them can be different.

The automated diagnostic system provides continuous data on the state of the equipment and serves to prevent a possible accident. It also serves to compile a technical data bank, which, in turn, can be used to determine whether the condition of the pump parts is at the required level or not $[8,9]$.

The developed systems must ensure high accuracy in determining the recorded parameters and be able to process the obtained data $[10,11]$. The number of installed sensors allows you to collect the necessary information to update the database and warn about possible emergency situations in the scientific equipment. The recorded data was selected taking into account the used control system of the pumping unit - SCADA (figure 3).

The norms recommended by the diagnostic system are shown in figure 3 .

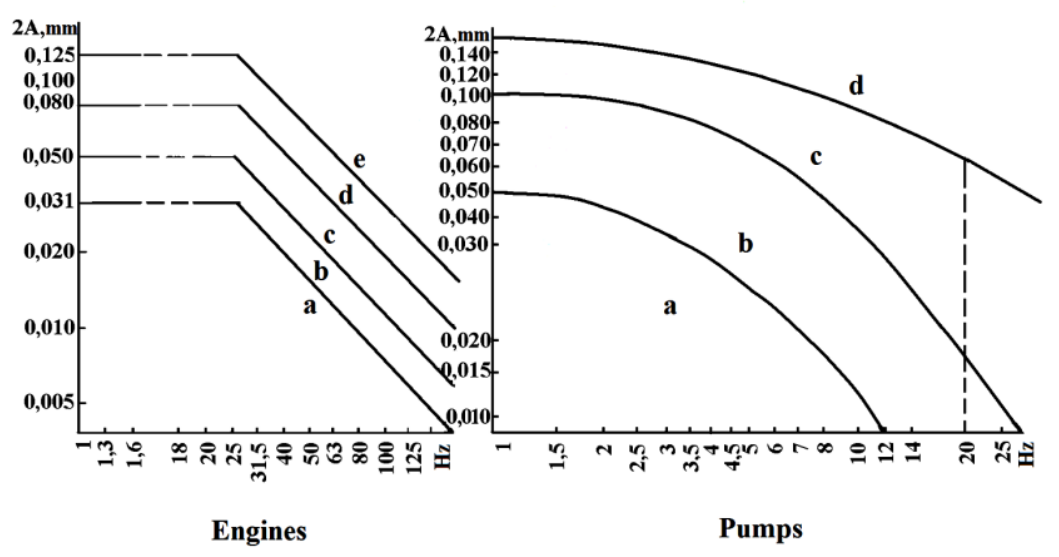

Fig. 3. Standards for assessing the vibrational state of the pump unit: $a$ - fine; $b$ - well; $c$ - satisfactorily; $\mathrm{d}$ - unsatisfactory; e - unacceptable.

The reliability function of scientific equipment is determined as a result of taking into account a number of factors: the external environment, technological properties of systems, operational requirements, etc.

The obtained reliability indicators of the objects are compared with the reliability indicators required by the technical conditions $[1,2]$. 


$$
P_{\text {estimated }} \geq P_{\text {reguired }}
$$

where $P_{\text {estimated }}$ is the calculated value of the lower bound of the estimation of the probability of no-failure operation;

$P_{\text {required }}$ is the required value of the probability of no-failure operation.

When the condition (2) is not met, the reliability requirement is considered unfulfilled and it is necessary to carry out the revision of the PU, which will ensure the fulfillment of this ratio.

When evaluating parametric reliability, the reliability condition is written:

$$
P \geq E
$$

where $P-$ is the potential ability of the structure to withstand external influences throughout the entire life of the pump unit;

$E$ - external influence.

Inequality (3) can express the condition for the reliability of the pump unit in the limit state (stability, bearing capacity, deformations, etc.).

Function $E$ can express both external influences and deformations arising from them. In the general case, all calculations of the pump unit, ensuring its reliable operation, can be reduced to equality

$$
v=Y_{1}-Y_{2} \geq 0
$$

where $Y_{l}$-is the sum of internal factors characterizing the bearing capacity (limit of permissible deformation, etc.); $Y_{2}-$ is the sum of external factors characterizing the acting loads with their most unfavorable combination

Then the reliability of the pump unit is determined by the probability of values greater than zero

$$
P=P(v \geq 0)
$$

When the relation and the law of distribution of random variables xi are known, then the moments of distribution of random variables are established by the reliability indicator $P$.

When the ratio $Y=f\left(x_{1}, x_{2}, \ldots, x_{n}\right)$ and the distribution law of random variables $x_{i}$ are known, then the moments of distribution of random variables are set according to the reliability indicator - the probability $P$.

One of the most important reliability characteristics is the scientific equipment availability. Therefore, when performing production tasks for a PU, a significant place is occupied by the issues of a thorough analysis of the transition of the system from one state to another and the dynamics of changes in the availability factor of PU for each state.

Let us assume that during the execution of the task, the PU is in n states, the period of time spent in each of them $t_{i}=t_{K_{i}}-t_{H_{i}}$. Here $t_{H_{i}}$ and $t_{K_{i}}$ are the times of the beginning and end of being in the $t_{M}$ state.

The total time of normal operation of the pump unit can be determined as follows:

$$
T=\sum_{i=1}^{n} t_{M_{1}}+\sum_{t=1}^{n}\left(t_{K_{i}}-t_{H_{i}}\right) .
$$

The following equation can be used to estimate the unit availability at each time interval:

$$
\pi_{i}(t)+\left(\lambda_{i}+\mu_{i}\right) \pi_{i}(t)=\mu_{i}
$$


Here $\pi_{i}(t)$ - is the availability factor of the scientific equipment in the interval $\left[t_{H_{i}}, t_{K_{i}}\right]$; $\lambda_{i}$ and $\mu_{i}-$ the rates of failure and recovery in a given interval.

The general solution of differential equation (7) has the form [12]

$$
\pi_{i}(t)=\frac{\mu_{i}}{\lambda_{i}+\mu_{i}}+C e^{-\left(\lambda_{i}+\mu_{i}\right) t_{i}}
$$

Here $t_{i} \in\left[t_{H_{i}}, t_{K_{i}}\right]$.

To find the constant $\mathrm{C}$ for each state, you can use the following initial conditions:

$$
t_{i}=t_{H_{i}}, \pi_{i}\left(t_{i}\right)=\pi_{i}\left(t_{H_{i}}\right),(i=1,2, \ldots, n)
$$

Using the initial conditions from the general solution (7), we obtain

$$
\pi_{i}\left(t_{H_{i}}\right)=\frac{\mu_{i}}{\lambda_{i}+\mu_{i}}+C e^{-\left(\lambda_{i}+\mu_{i}\right) t_{H i}}
$$

From where

$$
C=\frac{\pi_{i}\left(t_{H_{i}}\right)\left(\lambda_{i}+\mu_{i}\right)-\mu_{i}}{\lambda_{i}+\mu_{i}} e^{\left(\lambda_{i}+\mu_{i}\right) t_{H_{i}}}
$$

Substituting (11) into (8), we obtain particular solutions of differential equations in the following form:

$$
\pi_{i}(t)=\frac{\mu_{i}}{\lambda_{i}+\mu_{i}}+\frac{\pi_{i}\left(t_{H_{i}}\right)\left(\lambda_{i}+\mu_{i}\right)-\mu_{i}}{\lambda_{i}+\mu_{i}} e^{\left(\lambda_{i}+\mu_{i}\right)\left(t_{H_{i}}-t_{i}\right)}
$$

In expression (12) $t_{H_{i}}-t_{i} \leq 0$, therefore, when $t_{i} \rightarrow t_{K_{i}}$, the value $\pi_{i}\left(t_{i}\right)$ decreases.

In the study of the availability coefficient of the pumping unit, the following two cases are encountered:

1) the availability factor of the pump unit $\pi_{i}\left(t_{i}\right)$ is equal to the availability factor at the end of the previous time interval, i.e. $\pi_{i}\left(t_{i}\right)=\pi_{i-1}\left(t_{K_{i-1}}\right)$;

2) the availability factor of the pump unit $\pi_{i}\left(t_{i}\right)$ is not equal to the availability factor at the end of the previous time interval, i.e. $\pi_{i}\left(t_{i}\right)=\pi_{i-1}\left(t_{K_{i-1}}\right)+\alpha_{i}$, where $\alpha_{i}$ are the jumps in the availability factors during the transition from one state $\alpha_{i}$ to another. In this case, if, taking into account the proposed methodology, it will be possible to reduce the absolute value to zero, then the study can be continued as in the first case, otherwise additional research is required to study $\alpha_{i}$.

Consider the implementation algorithms of the first case. In this case, instead of the initial conditions (6), it is sufficient to use only one initial condition set for the first time interval:

$$
t_{i}=t_{H_{i}}, \pi_{i}\left(t_{i}\right)=\pi_{i}\left(t_{H_{i}}\right)
$$

For the remaining time intervals, $\pi_{i}\left(t_{K_{i}}\right)$ it can be determined from the initial conditions 


$$
t_{i}=t_{H_{i}}, \pi_{i}\left(t_{i}\right)=\pi_{i-1}\left(t_{H_{i}}\right),(i=2, \ldots, n) \text {, given that } \pi_{y_{i+1}}\left(t_{H_{i+1}}\right)=\pi_{i}\left(t_{K_{i}}\right) .
$$

The operational availability factors for each time interval can be obtained by calculation sequentially, in ascending order of the numbers of time intervals. This algorithm greatly facilitates the process of updating the equipment availability factor for the entire period of operation.

Similarly to this technique, it is possible to carry out a multi-stage examination of the state of the PU according to other reliability characteristics [14].

The unit is a collection of individual nodes, the state of which determines the performance of the entire object. The superposition of vibroacoustic fields generated by them creates a complex wave pattern characterized by spectral distribution. Separation of signals of certain frequencies allows calculating diagnostic parameters and eliminating the influence of extraneous noise on their values.

The construction of a model of the technical state of PU is to create a list of recognizable states with a description of their diagnostic vectors and the determination of informative parameters that have boundaries of normal and abnormal states.

To diagnose PU, it is necessary to have two types of information that allows, on the one hand, to determine the boundaries of normal and abnormal states, and on the other hand, to determine the real values of the parameters characterizing the state of PU during the period of its examination.

The first type is an individual set of technical characteristics. It includes:

- pump and motor diagnostics parameters;

- frequency of rotation of the PU shaft;

- types of bearings and their identification numbers;

- the number of pump impellers and the number of blades on each of them.

Spectra of vibroacoustic signals are another type of input information. Changes in their amplitudes at characteristic frequencies indicate deviations in the operation of the unit. Since the values of the parameters are individual for each PU, a method of relative measurements should be chosen for those of them that cannot be estimated in absolute values.

The main diagnostic parameter, regulated by GOST, is the value of vibration velocity. The analysis of the spectra showed that the greatest contribution to the integral value is made by harmonics and subharmonics of the shaft rotation frequency $[5,10]$.

The experience of experimental studies has shown that free flow surfaces are established in the water supply structures of the pumping stations, which sharply change the depths and velocities downstream on a rather short section of the path and, as a result, increase the forces of resistance to motion. Therefore, in addition to the resistances due to the pressure difference and friction, in accordance with the principles of hydromechanics, it is recommended to take into account the forces of vortex formation or flow and the forces of inertia in the flow path of the pumps $[15,16]$.

If the characteristics are independent, then the probability $P_{1}$ for the $j$-th mode anomaly is calculated as follows

$$
P_{1 j}=P\left(D_{0}\right) \prod_{i=1}^{N} \int_{H_{j i}}^{B_{j i}} f\left(\frac{\stackrel{\circ}{i}_{i}}{D_{0}}\right) d \stackrel{\circ}{\chi_{i}}
$$

The probability of missing the $\mathrm{j}$-th anomaly, respectively, will be equal to

$$
P_{2 j}=P\left(D_{j}\right) \prod_{i=1}^{N} \int_{H_{0 i}}^{B_{0 i}} f\left(\frac{\stackrel{\circ}{\chi}_{i}}{D_{0}}\right) d \stackrel{\circ}{\chi_{i}}
$$


where $D_{0}$ is the probability density of the normal state;

$D_{j}$ is the probability density of the anomalous state.

The false alarm probability for $M$ anomalies is

$$
P_{1}=P\left(D_{0}\right) \sum_{j=1}^{M} \prod_{i=1}^{N} \int_{H_{j i}}^{B_{j i}} f\left(\frac{\stackrel{\circ}{\chi}_{i}}{D_{0}}\right) d \stackrel{\circ}{\chi}_{i}
$$

here $P\left(D_{j}\right)$ is the prior probability of the $j$-th state.

In the general case, the parameters are not independent; therefore, to apply formulas (14) - (16) in estimating the error probabilities, additional orthogonalizing transformations are required. In formula (16) $\mathrm{L}$ is the number of spectral lines.

The accelerating vibration characteristics of the scientific equipment are recorded in the process from the moment of start-up to reaching the steady state. The main feature of the accelerating characteristics of the PU is a short time interval from the moment the electric motor is switched on until the rotor system is fully accelerated. Studies have shown that the acceleration time of an PU under load is usually less than $3 \mathrm{~s}[17,18]$. Examples of overclocking characteristics for PU are shown in Figure 4.

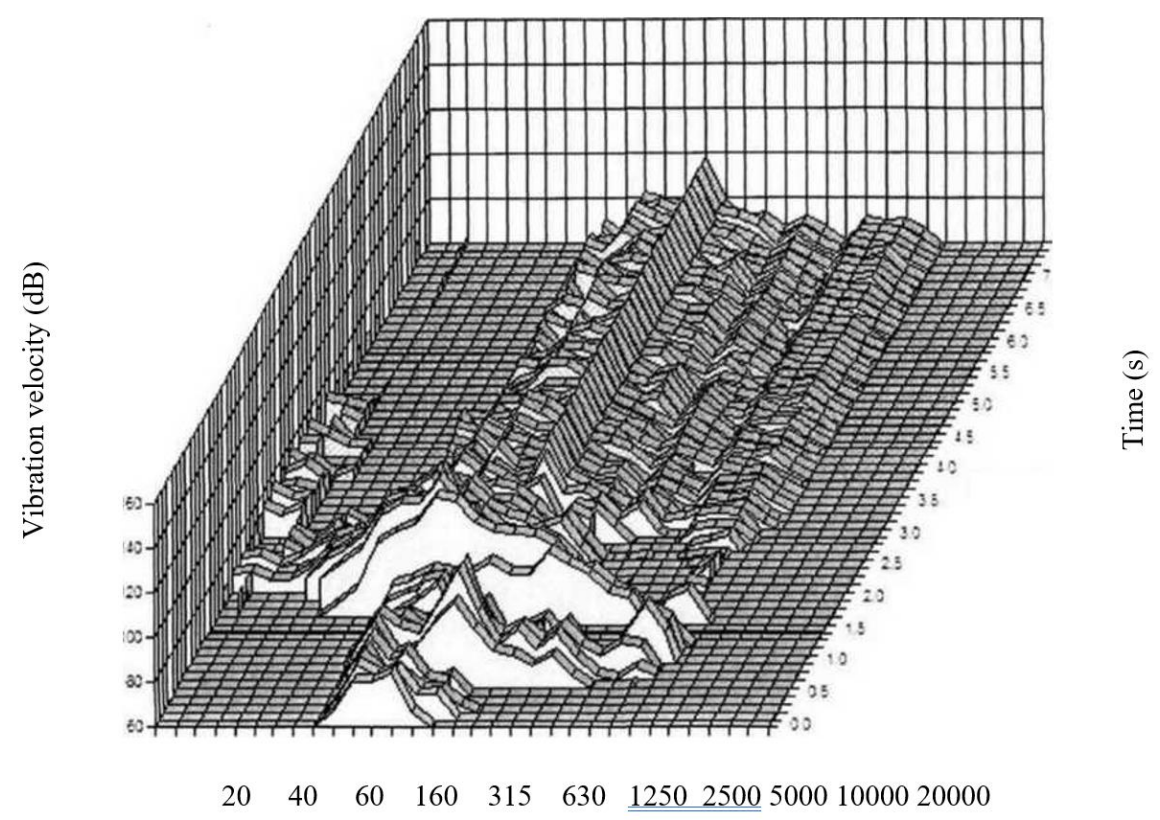

Fig. 4. Multispectrum of logarithmic levels of vibration velocity during acceleration of PU.

Defects of impeller blades caused by wear, deformation, as well as different distances between the blades can cause discrete vibration components at the revolving frequency, its first and second harmonics, blade frequency and associated lateral frequency components.

In Figure 5 shows a photograph of the monitor screen with the image of vibration parameters of PU № 3 of the PS "Narpai" $[15,18]$. The photo shows the vibration velocity diagrams of bearings with a characteristic sharp change in the parameters of low-frequency vibration vibrations. 


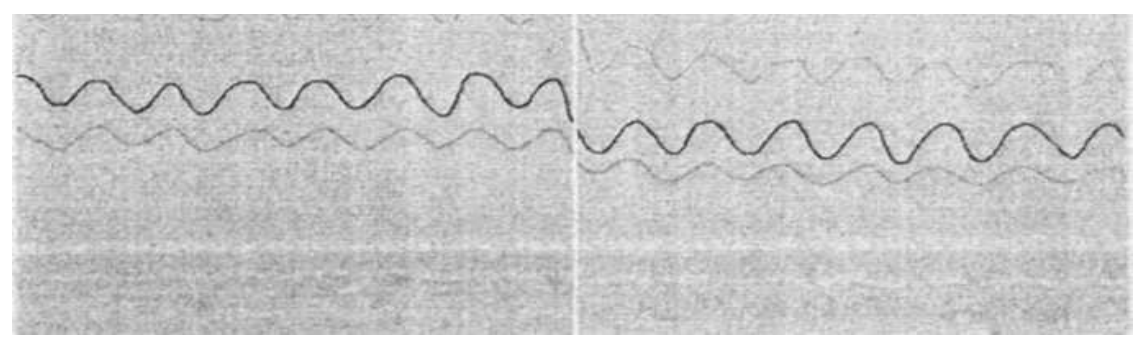

Fig. 5. Diagrams of vibration velocity of bearings on a standard control system PU № 3 "Narpai"

The performed analysis allows us to conclude that low-frequency fluctuations in the vibration intensity of the PU are associated with changes in the parameters of the units.

\section{Conclusion}

1. To assess the technical condition of scientific equipment, the proposed diagnostic systems allow obtaining and storing more data on vibration of scientific equipment and analyzing the data obtained for each scientific equipment.

2 . As a result of the implementation of the proposed diagnostic system, the methods of studying the parameters in time during the operation of the scientific equipment were used to prevent accidents resulting from a technological failure, based on the information received about changes in the technical state of the scientific equipment depending on the level of vibration indicators.

3. A method for calculating the reliability of scientific equipment is proposed. Operation modes can be controlled with the use of various scientific equipment availability factors.

The described technique allows you to accurately determine the malfunction that causes increased vibration of the unit. In addition, the software is designed in such a way that the technique can be tested on test signals (or with the introduction of known parameter values). The initial stage of searching for defects can be varied by changing the threshold values of probable conditions, as well as the values of the settings for each parameter.

\section{References}

1. O. Ya. Glovatsky and Ergashev R. R. Investigations of the reliability and condition of the system of machine water-lifting // Materials of the international scientific and technical. conf. "Modern problems of mechanics", pp. 77-80, 2009

2. B. Kh. Abzalov and Glovatsky O. Ya. New methods for assessing the technical condition of reclamation pumping stations of machine water-lifting systems // Materials of the scientific-practical conference "Development of water management and land reclamation in the Republic of Uzbekistan" Proceedings of SANIIRI. pp. 100104, 2006

3. N. A. Muminov, To the calculation of the reliability of flexible production complexes // Journal "Problems of Mechanical Engineering and Automation". M.; Budapest, . Issue 15. pp. 57-60, 1987

4. O. Ya. Glovatsky, Ergashev R. R. and Rustamov Sh. R. New methods for increasing the reliability of the quantitative assessment of the reliability of the operation of objects of systems of machine lifting // International scientific-practical conference "Land reclamation and problems of restoration of agriculture in Russia" (Kostyakov readings) (VNIIGiM, Russian Agricultural Academy. Moscow) pp. 372-376, 2013 
5. O. Ya. Glovatsky and Bekchanov F. A. Improving the methods for diagnosing pumps of large hydraulic systems // Journal of Hydrotechnics, No. 2 (55), Science and Technology, St. Petersburg pp.70-73, 2019

6. F. Bekchanov, Ergashev R., Mavlanov T. and Glovatskiy O. Mathematical model of vibrating air pump unit // XXII International Scientific Conference on Advanced in Civil Engineering / construction the formation of living environment, April 18-21, 2019

7. F. A. Bekchanov, New methods for diagnosing pumps hydrotechnical systems. International journal for innovative research in multidisciplinary fields. Vol 4, Issue 10, Oct-2018. pp. 367-373 (Research Bib No. 14 IF-5.6, Scientific Journal Impact Factor No. 23 IF-6.497), 2018

8. O.Ya. Glovatsky, Ergashev R.R., Bekchanov F.A., Rustamov Sh.R. Assessment of the technical condition and availability of pumping units. // International Agricultural Engineering, Scientific and Technical Journal, Almaaty-2012. pp.64-67.

9. R.R. Ergashev, Bekchanov F.A., Nasyrova N.R. Diagnostic tests of vertical pumps. // Materials of the international scientific-practical conference "Ways to improve the efficiency of irrigated agriculture" - Novocherkassk, No. 3 (59), pp.31-36, 2015

10. R. R. Ergashev, Glovatsky O. Ya., Bekchanov F. A. and Nasyrova N. R. New diagnostic methods for large vertical pumping units. // Materials of the republican scientific and technical conference "Improving the exploitation and saving of water resources" -Toshkent.pp.397-402, 2015

11. O.Ya. Glovatsky, Ergashev R.R., Bekchanov F.A. Diagnosis analysis of pumping units of the Jizzakh head pumping station. // "Irrigatsiya va melioratsiya" journal No. 3 (9). Toshkent. 2017. Pp. $32-34$ (05.00.00; No. 22).

12. Methodical recommendations MR 125-02-95. Rules for drawing up design schemes and determining the parameters of loading of structural elements with identified defects / TsNIITMASH, 1995, 24 p.

13. E. Kan, Mukhammadiev M., Ikramov N. and Majidov T. Full-scale testing of the pump unit with a frequency converter IOP Conference Series: Materials Science and Engineering vol 883 (IOP Publishing Ltd) p 012112, 2020

14. O.Ya. Glovatsky, R.R. Ergashev, N.R. Nasyrova, F.A. Bekchanov. The program to ensure the reliability of pumping units // No. DGU 03969. Certificate of official registration of the program for electronic computers. 09.09.2016

15. A. S. Gazaryan, et al. Improvement of technological modes of pumping stations in conditions of unsteady fluid movement // Scientific and practical journal "Ways to improve the efficiency of irrigated agriculture" - Novocherkassk, No. 4 (76), 2019. - p. 137-142.

16. O. Glovatsky, Azizov O., Shamayramov M., Bekchanov F., Gazaryan A. and Ismoilov N. Diagnostic tests of vertical pumps modernized pump stations IOP Conference Series: Materials Science and Engineering vol 883 (IOP Publishing Ltd) p 012032, 2020

17. O. Ya. Glovatsky, N.R. Nasyrova, Operation and maintenance of the technical condition of pumping station equipment, diagnostics // Textbook / Course of lectures and practical exercises. UNDP, UNESCO. T. $2019.130 \mathrm{p}$.

18. Sh. Rustamov, N. Nasirova, Constructive peculiarities of modernized centrifugal pump // European science review, № 3-4, 2018. Vienna. pp-278-280. 